\title{
Functional connectivity of reward processing in the brain
}

\section{Estela Camara ${ }^{1,2}$, Antoni Rodriguez-Fornells ${ }^{2,3}$ andThomas F. Münte ${ }^{1,4 *}$}

\author{
Department of Neuropsychology, University of Magdeburg, Magdeburg, Germany \\ 2 Department of Ciencies Fisiològiques, University of Barcelona, Barcelona, Spain \\ 3 Institució Catalana de Recerca i Estudis Avançats, Barcelona, Spain \\ ${ }^{4}$ Center for Behavioral Brain Sciences, Magdeburg, Germany
}

\section{Edited by:}

Francisco Barcelo, University of Illes

Balears, Spain

\section{Reviewed by:}

Michael X. Cohen, University of

Amsterdam, The Netherlands

Markus Ullsperger, Max Planck

Institute for Neurological Research,

Germany

\section{*Correspondence:}

Thomas F. Münte, Department of Neuropsychology, University of

Magdeburg, Gebäude 24,

Universitätsplatz 2, 39106 Magdeburg,

Germany.

e-mail: thomas.muente@medizin. uni-magdeburg.de
Controversial results have been reported concerning the neural mechanisms involved in the processing of rewards and punishments. On the one hand, there is evidence suggesting that monetary gains and losses activate a similar fronto-subcortical network. On the other hand, results of recent studies imply that reward and punishment may engage distinct neural mechanisms. Using functional magnetic resonance imaging (fMRI) we investigated both regional and interregional functional connectivity patterns while participants performed a gambling task featuring unexpectedly high monetary gains and losses. Classical univariate statistical analysis showed that monetary gains and losses activated a similar fronto-striatallimbic network, in which main activation peaks were observed bilaterally in the ventral striatum. Functional connectivity analysis showed similar responses for gain and loss conditions in the insular cortex, the amygdala, and the hippocampus that correlated with the activity observed in the seed region ventral striatum, with the connectivity to the amygdala appearing more pronounced after losses. Larger functional connectivity was found to the medial orbitofrontal cortex for negative outcomes. The fact that different functional patterns were obtained with both analyses suggests that the brain activations observed in the classical univariate approach identifies the involvement of different functional networks in the current task. These results stress the importance of studying functional connectivity in addition to standard fMRI analysis in reward-related studies.

Keywords: reward, nucleus accumbens, connectivity, functional magnetic resonance imaging

\section{INTRODUCTION}

Bribing someone with cash or, alternatively, threatening him with a high penalty powerfully influences behavior. Expectations of both punishment and reward also have an impact on future decisions, since we try to increase the probability of the occurrence of positive reinforcement and to minimize the recurrence of adverse event (Daw et al., 2006).

The delineation of the neural circuits subserving the processing of rewards and punishments and their translation into action is therefore of great importance, but controversial findings have been reported. On the one hand, there is ample evidence indicating that monetary gains and losses activate a similar fronto-subcortical network but to a differential degree (Dreher, 2007; Gottfried et al., 2003; Marco-Pallares et al., 2007; Nieuwenhuis et al., 2005; Tom et al., 2007; van Veen et al., 2004). On the other hand, recent studies suggest that reward and punishment outcomes may be processed by different neural circuits (Frank et al., 2004; Wrase et al., 2007; Yacubian et al., 2006). For example, modeling work of Frank et al. (2004) distinguishes between two excitatory/inhibitory pathways in the basal ganglia, which show differential modulation during positive and negative reinforcement processing. Dopamine (DA) release is typically evoked by positive outcomes, and in turn increases the activity in the excitatory pathway and suppresses the activity in the inhibitory connection. In contrast, negative events are associated with dips in DA levels, and thus show opposite effects. Drawing on neuroimaging results, Yacubian et al. (2006) distinguished two systems involved in predictions made concerning either possible gains or losses: By this account the ventral striatum generates predictions based on possible gains and compares these to actual outcomes, whereas the amygdala is involved in the prediction of possible losses, again comparing these to actual outcomes. A crucial factor in the processing of positive and negative feedback is the neurotransmitter DA which is underscored by recent research on the possible contribution of genetic variability in the DA system to interindividual differences in feedback and reward processing (Cohen et al., 2005; Frank et al., 2007; Klein et al., 2007; Yacubian et al., 2007).

Although there is a large body of neuroimaging literature concerning the regions involved in the processing of rewards and punishments, the picture provided is rather static. In the present communication we therefore investigate the functional connectivity patterns between different cortical and subcortical regions in response to monetary gains and losses. Indeed, a complete understanding of reward processing requires not only to identify the activated brain regions, but also to distinguish how these regions flexibly interact in response to different outcomes. Previous results addressing other cognitive processes have shown that analyses of functional connectivity are not redundant when compared with standard analyses of brain activation changes (Buchsbaum et al., 2005; Fiebach and Schubotz, 2006; Gazzaley et al., 2004; Ranganath et al., 2005; Rissman et al., 2004). In particular, different functional connectivity patterns may reveal different brain networks that in 
turn might mediate various aspects of behavior. For instance, Cohen et al. (2005) demonstrated an increase of the estimated functional connectivity between the anterior cingulate cortex (ACC) and the nucleus accumbens (NAcc), when comparing high-risk vs. low-risk gambling decisions. Klein et al. (2007) in a feedback-based learning task have shown a dynamically changing functional connectivity pattern between the dorsal ACC, the hippocampal formation, and the ventral striatum that enables the learning of stimulus-reward associations across the task. Cohen et al. (2008) also reported recently that the microstructural properties of white matter tracts connecting the amygdala to the hippocampus, orbitofrontal cortex (OFC), and the ventral striatum predicted functional connectivity derived from functional magnetic resonance imaging (fMRI) time series and participants' behavior following both positive and negative feedback in a reversal learning task.

Which regions might interact in the processing of reward? The ventral striatum has been proposed to be involved in the selection of appropriate responses and the modulation of goal-directed behavior (Berridge and Robinson, 1998; Kelley and Berridge, 2002). This region has shown increased activation in the presence of positive reward outcomes (monetary gains) when compared to negative outcomes (monetary losses) in several studies (Delgado et al., 2000, 2003; May et al., 2004; Riba et al., 2008). More importantly, studies by Tom et al. (2007) have recently shown that activation in the ventral striatum decreased as the size of the potential loss increased. The ventral striatum receives synaptic inputs from the OFC, dorsolateral prefrontal cortex, and limbic structures such as hippocampus and amygdala (Groenewegen et al., 1999). It is also the target of dense dopaminergic projections originating in the ventral tegmental area (VTA) (Voorn et al., 1986). The VTA has been repeatedly associated to learning and motivation (Schultz, 2007). Therefore the ventral striatum is a key candidate in the study of functional connectivity in the context of reward processing.

With this aim, healthy volunteers were involved in a simple gambling task modified from previous studies (Gehring and Willoughby, 2002; Marco-Pallares et al., 2008; Riba et al., 2008). In this task unexpected and large monetary gains and losses (henceforth boost trials) occurred infrequently in addition to frequent gain and loss trials of smaller magnitudes. We used the "beta series correlation" method proposed by Rissman et al. (2004) to examine event-related changes in whole-brain functional connectivity with the ventral striatum as a seed region and to compare gain and loss functional connectivity patterns. If two regions are functionally interacting in response to a specific event, the fluctuation of blood oxygen level dependent (BOLD) activity of both regions across trials should be correlated. We predict that the role of reward and loss outcomes might be functionally differentiated by examining patterns of brain connectivity. To our knowledge, no other study has directly compared functional connectivity between reward and loss outcomes.

\section{MATERIALS AND METHODS PARTICIPANTS}

Seventeen young adult students [10 women, 21.6 $\pm 2.6(\mathrm{SD})$ mean age] from the University of Barcelona participated in the study. All participants were healthy, right-handed native Spanish speakers with no history of neurological or psychiatric episodes. They all gave written informed consent to a protocol approved by the University of Barcelona ethics committee.

\section{TASK DESIGN}

Several important modifications were made to the monetary gambling task designed by Gehring and Willoughby (2002). In the standard trials $(80 \%)$ a warning signal was presented (“*”; $500 \mathrm{~ms}$ duration) followed by the presentation of two numbers ( 5 and 25) displayed in white against a black background in the two possible combinations, [525] or [25 5]. Participants had to select one of the two numbers by pressing a spatially corresponding button with the left or right index finger. One second after the choice, one of the numbers turned green and the other turned red. If the number selected by the participant changed to red, the participant incurred a loss of the corresponding amount of money in Euro cent. In contrast, if the number turned into green, this indicated a gain.

In addition to the standard trials described above, two additional conditions were created in order to assess brain responses to unexpected rewards and losses. In $10 \%$ of the trials ("boost unexpected trials"), an unexpectedly large gain or loss occurred. Independently of the chosen item (either 5 or 25) the feedback turned into 125 ( $125 €$ cents), again having either green or red color indicating wins or losses. To control for the fact that boost trials were both, large and unexpected, in an additional 10\% of the trials ("similar unexpected") the chosen number turned to either 7 (instead of 5) or 27 (instead of 25). While these trials were unexpected, the magnitude of the gain or loss was virtually unchanged. The four possible outcomes for the standard trials ([25 5] [5 25] [5 25] [25 5]), and the respective versions for the unexpected similar trials and for the unexpected boost trials were presented in random order. Additionally, each run included 12 randomized fixation trials that lasted $20 \mathrm{~s}$.

Participants were provided with an initial $10 €$ sum and were encouraged to win as much as possible. They had performed in a similar event-related potential task (without unexpected trials) several weeks to months earlier and thus were familiar with the task in general. They were informed about the potential occurrence of unexpected trials. The experiment comprised four blocks of 140 trials each. These combinations were counterbalanced by condition, making the statistically expected outcome 0 on each trial in order to avoid confounds of differential probability of gains or losses. At the end of each run, participants were informed about the accumulated amount of money. At the end of the experiment, participants were paid the final amount obtained.

One of the attributes that boosts the impact of reward is the degree of uncertainty that exists in the estimation of the action's value. Reward information becomes more relevant as the uncertainty of the reward prediction and the magnitude of the outcome increases (Fiorillo et al., 2003; Schultz et al., 1997). Indeed, two recent fMRI studies have provided evidence that the NAcc response is larger to less likely rewards (Abler et al., 2007; Yacubian et al., 2006). Moreover, dynamic changes in functional connectivity patterns have been shown over the time course of an experiment indicating learning of stimulus-reward associations (Klein et al., 2007). We therefore assumed that the reward-related activations would be more pronounced and constant across the experiment for the unexpected boost trials which is why we primarily focus on this class of trials. To check whether the results obtained for the boost trials can be generalized to the standard condition [Gain/Loss $(5 / 25)]$ or instead reflect only surprise, functional and connectivity gain and loss-related patterns were assessed for standard trials as well. To compare these trials, we created four analogous functional 
contrasts: Gain (125) vs. fixation, Gain $(5+25)$ vs. fixation, Loss (125) vs. fixation and Loss $(5+25)$ vs. fixation. Second, brain regions responding selectively to gains and losses were defined by the gain vs. loss contrast in the boost and the standard trials reflecting the effect of valence in the expected and unexpected condition.

\section{fMRI ACQUISITION}

fMRI data was collected using a 3T whole-body MRI scanner (Siemens Magnetom Trio, Erlangen, Germany). Visual images were back-projected onto a screen using an LED-projector and participants viewed the images through a mirror on the head coil. Magnet compatible response buttons were used. Conventional high-resolution structural images [magnetization-prepared, rapidacquired gradient echoes sequence, 192 slice sagittal, TR $=2500 \mathrm{~ms}$, $\mathrm{TE}=4.77 \mathrm{~ms}, \mathrm{TI}=1100 \mathrm{~ms}$, flip angle $=7^{\circ}, 1 \mathrm{~mm}$ thickness (isotropic voxels)] were followed by functional images sensitive to BOLD contrast (echo planar T2*-weighted gradient echo sequence, $\mathrm{TR}=2000 \mathrm{~ms}, \mathrm{TE}=30 \mathrm{~ms}$, flip angle $=80^{\circ}$ ). Each functional run consisted of 336 sequential whole-brain volumes comprising 32 axial slices aligned to the plane intersecting the anterior and posterior commissures, $3.5 \mathrm{~mm}$ in-plane resolution, $4 \mathrm{~mm}$ thickness, no gap, positioned to cover all but the most superior region of the brain and the cerebellum.

\section{MRI ANALYSIS}

Functional images were analyzed using standard procedures implemented in the Statistical Parameter Mapping software (SPM2, http://www.fil.ion.ucl.ac.uk/spm). First, functional volumes were phase shifted in time with reference to the first slice to minimize purely acquisition-dependent signal-variations across slices. Head-movement artefacts were corrected based on an affine rigid body transformation, where the reference volume was the first image of the first run (e.g., Friston et al., 1996). Functional data was then spatially smoothed with an $8 \mathrm{~mm}$ full-width half-maximum (FWHM) isotropic Gaussian Kernel.

For the group-level analyses, realigned functional data was averaged and the mean functional image was normalized to a standard stereotactic space using the EPI derived MNI template (ICBM 152, Montreal Neurological Institute) provided by SPM2. After an initial 12-parameter affine transformation, an iterative non-linear normalization was applied using discrete cosine basis functions by which brain warps are expanded in SPM2 (Ashburner and Friston, 1999). Resulting normalization parameters derived for the mean image were applied to the whole functional set. Finally, functional EPI volumes were resampled into $4 \mathrm{~mm}$ cubic voxels and then spatially smoothed with an $8 \mathrm{~mm}$ FWHM isotropic Gaussian Kernel to minimize effects of inter-subject anatomical differences. Notice, that all statistical analyses, with the exception of the group statistics in the functional connectivity analysis (see below) were performed in native space (i.e., without spatial normalization).

\section{Univariate fMRI analysis}

The statistical evaluation of our data was based on a least-square estimation using the general linear model by modeling the different conditions with a regressor waveform convolved with a canonical hemodynamic response function (Friston et al., 1998). Thus, an event-related design matrix was created including the conditions of interest: Gain 5, Gain 25, Gain 7/27, Gain 125, Loss 5, Loss 25,
Loss $7 / 27$, Loss 125 , and fixation. Both native and normalized data was high-pass filtered (to a maximum of $1 / 90 \mathrm{~Hz}$ ), and serial autocorrelations were estimated using an autoregressive model $[\mathrm{AR}(1)$ model]. Resulting estimates were used for non-sphericity correction during the model estimation. Confounding effects in global mean were removed by proportional scaling, and signal-correlated motion effects were minimized by including the estimated movement parameters.

\section{Region of interest analysis}

Our approach for identifying functional networks requires the definition of an a priori region of interest (ROI), which is then used to determine which voxels throughout the whole-brain are functionally interacting with the selected ROI. Given the strong evidence implicating the ventral striatum in the processing rewards and losses, functional ROIs were defined as the largest cluster of connected voxels which were more strongly activated for gains than losses $(P<0.01$ uncorrected, 20 voxels spatial extent, restricted to the ventral striatum). In those participants for whom the activation extended beyond the ventral striatum the threshold was increased to $P<0.001$ or $P<0.0001$ in order to restrict the seed region to the ventral striatum (Figure 3 ).

\section{Functional connectivity analysis}

Functional connectivity analysis was performed using the method proposed by Rissman et al. (2004) using the parameter estimates obtained in the context of the general linear model. The beta series bivariate method uses a standard general linear model approach but adapts the model in such a way that separate beta values (general linear model parameter estimates) are obtained in order to modulate activation changes for each component of each individual trial. With this approach, a series of parameter estimates can be extracted from a seed region, correlated across the brain to identify specific networks.

The analysis was based on the hypothesis that if different regions are involved in a network, strongly correlated activity patterns should be observed among them and functional brain connections can thus be inferred. In particular, each specific trial was modeled as an independent covariate in the study design matrix, which allowed the assessment of a trial-to-trial parameter estimate for each condition. For each participant, the parameter estimates series obtained were sorted by conditions. For the conditions of interest (standard loss, boost loss, standard gain, and boost gain), the corresponding parameter estimates were averaged across all voxels of the left and right NAcc ROI. Thereafter, individual native space correlation maps were generated for each participant and condition by correlating the seed region with the beta series of each voxel in the whole-brain. To allow statistical inferences to be made based on the correlation magnitude, an arc-hyperbolic tangent transform was applied to normalize the correlation coefficients, which were then z-transformed. The z-transformed correlation maps were spatially smoothed with an $8 \mathrm{~mm}$ FWHM) isotropic Gaussian Kernel to minimize effects of inter-subject anatomical differences. The resulting condition-specific connectivity maps were then normalized by applying the corresponding normalization parameters, which had been computed earlier. The individual contrast images were entered into a second-level analysis employing a one-sample $t$-test using a random effects analysis within the general linear model in order to characterize the networks involved in processing gains and losses. 
Maps thresholded at $P<0.00001$, uncorrected for multiple comparisons, were used for further discussion. Thereafter, correlation maps were compared between the gain and loss contrast at the group-level applying a paired samples $t$-test, using a $P<0.001$ uncorrected for multiple comparison threshold.

Finally, under the assumption that functional interactions between brain regions should reflect differential functional brain activations, we investigated how the differential connectivity profile observed between the gain and loss condition interacted with the functional activation pattern: group-level standard parameter estimates were averaged separately by condition and compared with each other in the statistically significant connectivity cluster. The connectivity cluster applied for testing the expected interaction was defined under the differential connectivity pattern, using a $P<0.05$ (uncorrected for multiple comparisons) threshold.

\section{RESULTS}

UNIVARIATE ANALYSIS FOR GAIN AND LOSS TRIALS

In the present gambling task, we focus our analysis on the unexpected gain and loss boost trials. Standard functional univariate analysis was performed in order to compare the overall pattern of activity for gains and losses. This analysis revealed a very similar fronto-subcortical-parietal network when monetary gains or losses were incurred (see Tables 1 and 2). To summarize the tabulated

Table 1 | Main effects for gains and losses in the standard functional analysis for the boost trials.

Reward-related brain regions

Stereotactic coordinates

in boost trials

\begin{tabular}{llllll}
\cline { 2 - 3 } & $X$ & $Y$ & $Z$ & $T$ peak
\end{tabular}

\section{GAIN (125) VS. FIXATION}

$R$ caudate

L caudate

$\mathrm{R}$ cerebellum

L cerebellum

Cuneus

R DLPC

L FG/PHG

pre-SMA/ACC

$R$ ant INS

$\mathrm{R}$ IPL

$\mathrm{LIPL}$

Mesencephalon

$\mathrm{R}$ thalamus

$L$ thalamus

$R$ ventral striatum

$\mathrm{L}$ ventral striatum

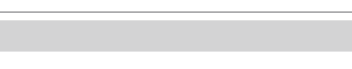

$\begin{array}{rrr} & 8 & \\ & -4 & 1 \\ & 20 & -7 \\ 46 & -24 & -7 \\ 37 & -4 & -5 \\ & 44 & 3 \\ 40 & -36 & -5 \\ 40 & 4 & 2 \\ & 36 & 2 \\ & 36 & -5 \\ & -36 & -5 \\ & -4 & -2 \\ & 4 & -1 \\ & -4 & -1 \\ & 8 & 1\end{array}$

\section{8}

16

$-72$

$-72$

$-50$

36

$-56$

24

20

$-56$

$-52$

$-28$

$-16$

$-12$

4

12

$x^{2}$

$\begin{array}{rr}4 & \\ 8 & 8.44 \\ -28 & 4.88 \\ -24 & 5.47 \\ -4 & 9.52 \\ 28 & 12.42 \\ -12 & 10.49 \\ 52 & 11.07 \\ -8 & 14.58 \\ 48 & 9.76 \\ 52 & 21.57 \\ -4 & 14.17 \\ 8 & 6.43 \\ 8 & 10.13 \\ -8 & 9.28 \\ -12 & 10.89 \\ & 12.24\end{array}$

LOSS (125) VS. FIXATION

\section{$R$ caudate}

L caudate

$\mathrm{R}$ cerebellum

L cerebellum

\section{Cuneus}

R DLPC

pre-SMA/ACC

L FG/PHG

R IPL

LIPL

$R$ ant INS

Mesencephalon

$R$ thalamus

$L$ thalamus

$R$ ventral striatum

L ventral striatum

$\begin{array}{rrr} & 8 & \\ & -4 & 16 \\ & 20 & -72 \\ 18 & -24 & -72 \\ 46 & -4 & -50 \\ & 44 & 36 \\ 37 & 4 & 24 \\ 40 & -36 & -56 \\ & 36 & -56 \\ & -36 & -52 \\ & 36 & 20 \\ & 0 & -28 \\ & 4 & -16 \\ & -4 & -12 \\ & 8 & 12 \\ & -16 & \end{array}$

$\begin{array}{rrr}8 & 4 & 8.44 \\ 16 & 8 & 4.88 \\ -72 & -28 & 5.47 \\ -72 & -24 & 9.52 \\ -50 & -4 & 12.42 \\ 36 & 28 & 10.49 \\ 24 & 52 & 14.58 \\ -56 & -12 & 11.07 \\ -56 & 48 & 21.57 \\ -52 & 52 & 14.17 \\ 20 & -8 & 9.76 \\ -28 & -8 & 6.17 \\ -16 & 8 & 10.13 \\ -12 & 8 & 9.28 \\ 4 & -8 & 10.89 \\ 12 & -12 & 12.24\end{array}$

MNI coordinates and T-value for the peak location in a particular identified anatomical cluster. $P<0.0001 ; 20$ voxels spatial extent uncorrected for multiple comparisons. Reported the P-value FWE-corrected P-value at voxel-level. BA, approximate Brodmann's area; L, left hemisphere; $R$, right hemisphere; IPL, inferior parietal lobe; SMA, supplementary motor area; ACC, anterior cingulate cortex; DLPC, dorso lateral prefrontal cortex; INS, insula; FG, fusiform gyrus; PHG, parahippocampal gyrus. 
Table 2 | Main effects for gains, losses, and gains vs. losses for standard trials in the classical functional analysis.

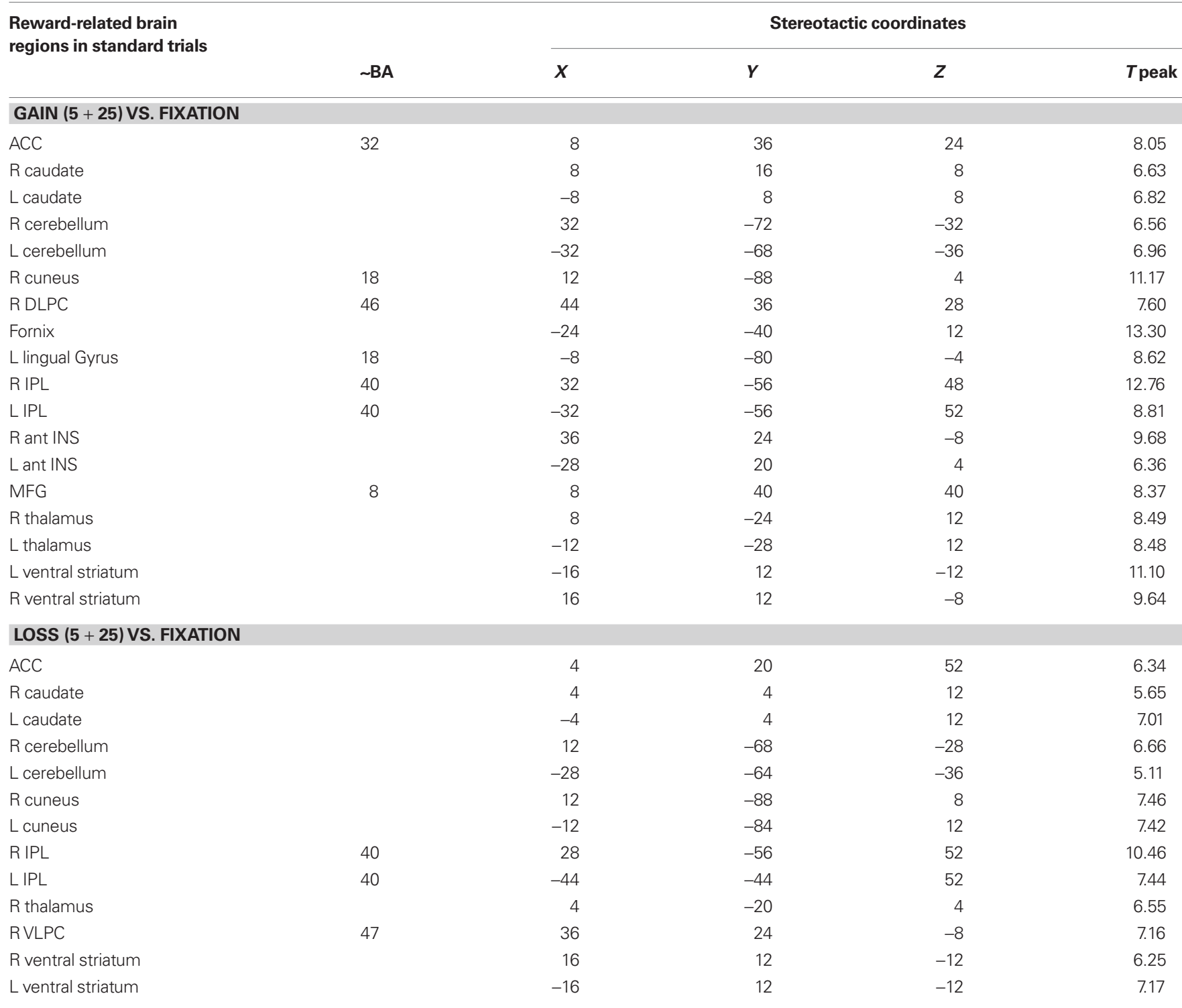

MNI coordinates and T-value for the peak location in a particular identified anatomical cluster. P< $0.0001 ; 20$ voxels spatial extent corrected for multiple corrections at cluster level. BA, approximate Brodmann's area; L, left hemisphere; $R$, right hemisphere; ant, anterior; IPL, inferior parietal lobe; SMA, supplementary motor area; ACC, anterior cingulate cortex; VLPC, ventral lateral prefrontal cortex; OFC, orbitofrontal cortex.

results, significant activations were observed in the cingulate cortex, the superior frontal cortex, the inferior parietal lobe, the insular cortex, parahippocampal regions, the thalamus, the caudate nuclei, the cerebellum, the cuneus, and the ventral striatum. Interestingly, mesencephalic activation was found in both the gain and loss condition in the boost trials but this region was not significant activated in the standard trials. Indeed, and consistent with previous studies, monetary gains elicited greater activation compared to loss trials. The gain vs. loss contrast [i.e., Gain (125) vs. Loss (125)] showed bilateral activation in the ventral striatum (NAcc) in all participants (peak activity, MNI coordinates, $x, y, z$, left hemisphere, $-16,4,-4, T=8.81, P<0.002$; right hemisphere, coordinates 16 , $8,-16, T=8.62, P<0.002 ; P$-value at FWE voxel-level corrected). In the standard contrast [i.e., Gain $(5+25)$ vs. Loss $(5+25)]$ a bilateral activation in the ventral striatum (NAcc) was found as well in all participants (peak activity, MNI coordinates, $x, y$, $z$, left hemisphere, $-16,4,-12, T=11.89, P<0.001$; right hemisphere, coordinates $16,8,-12, T=7.59, P<0.013 ; P$-value at FWE voxellevel corrected). Additionally, no significant differences were found in the inverse contrast (loss vs. gain trials), even after lowering the threshold to $P<0.05$ uncorrected. Overall, the analysis shows that positive and negative outcomes evoked very similar brain activity (see Figures 1 and 2).

\section{FUNCTIONAL CONNECTIVITY ANALYSIS}

Concerning functional connectivity, we used the ventral striatum (NAcc) identified above as a seed region (see Figure 3 ) to contrast gains vs. losses and to determine which brain regions significantly 


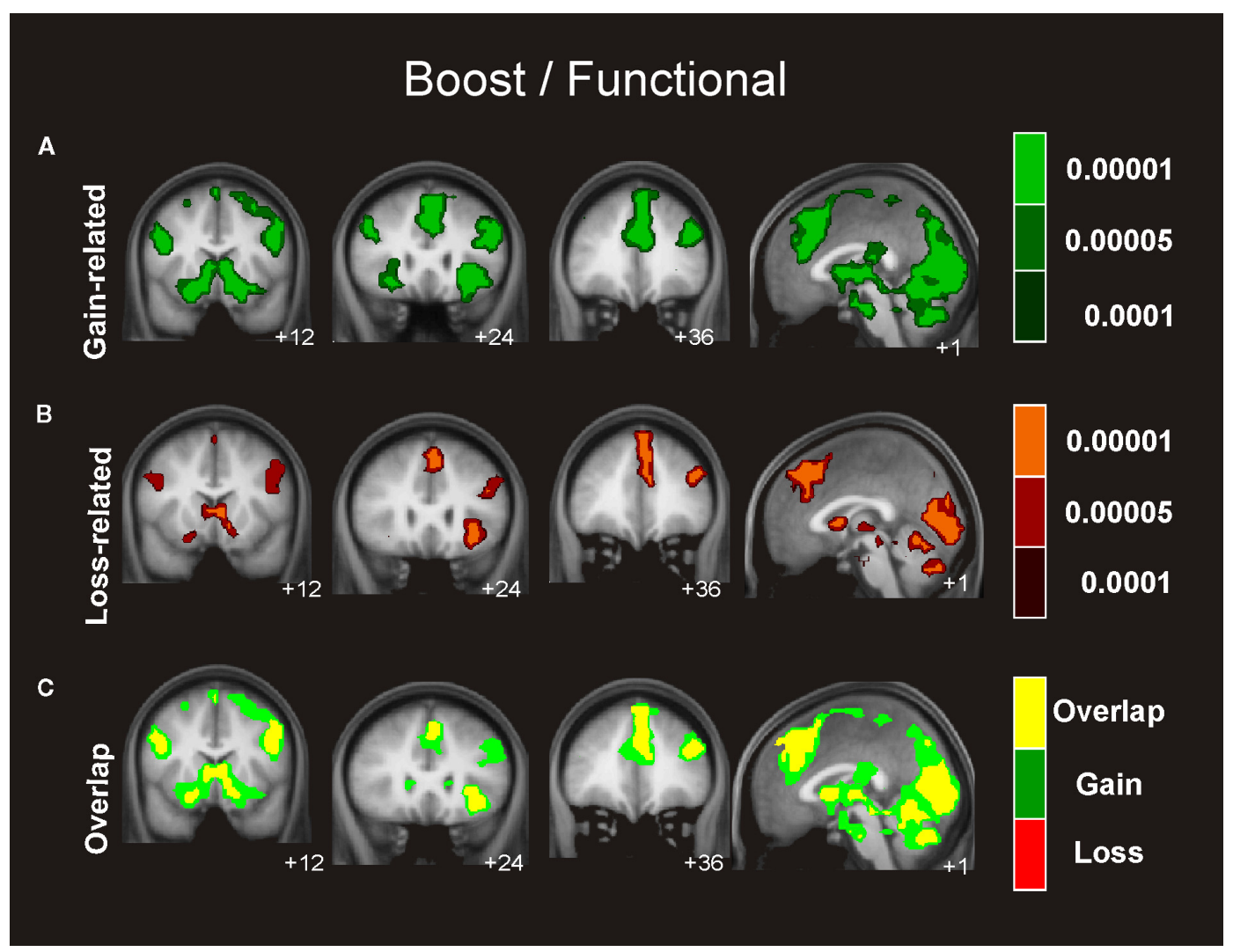

FIGURE 1 | Univariate fMRI analysis in the boost trials. Coronal and sagittal views of group average whole-brain univariate functional analysis superimposed on a group-averaged structural MRI image in standard stereotactic space ( $T$-score overlays). Shown are the gain vs. fixation contrast $(\mathbf{A})$ and the loss vs. fixation contrast (B) using different statistical thresholds $(P<0.0001 ; P<0.0005$; $P<0.001)$. Gain and loss patterns are simultaneously represented in $(\mathbf{C})$ : gain (green, $P<0.001$ ), loss (red, $P<0.001$ ), and conjunction gain $\cap$ loss (yellow, $P<0.001$ and $P<0.001$ ) correlated with activity in the ventral striatum. First, functional connectivity was examined separately for gains and losses and then tested for significant differences in connectivity between both conditions in boost and standard trials. The list of the reported functional connectivity interactions is presented in Tables 3 and 4.

Similar connectivity patterns were identified for the boost and the standard condition involving an extensive network of regions including the hippocampus, insular cortex, and OFC, which exhibited activity that correlated significantly with the activity seen in the ventral striatum in gain trials as well as in loss trials (see Figures 4 and 5 ).

We also investigated which regions showed a significant difference in correlation with the NAcc for gain > loss trials. Whereas this contrast revealed significant differences in connectivity neither for the boost nor for the standard condition, we found significant differences in the inverse contrast (i.e., loss $>$ gain) for the boost trials in the medial OFC (peak activity, $-8,24,-16$ ). To assess the functional consequences of the differential connectivity pattern group-level standard parameter estimates were compared between conditions in the statistically significant connectivity cluster. Indeed, the differential connectivity pattern also revealed a differential functional activation pattern when the gain and loss conditions were directly compared $[F(1,16)=5.3, P<0.036$, cluster size $=44$ voxels extent $]$.
A clear increase in BOLD signal was observed for gains, whereas a decrease was seen for losses (see Figure 6).

As visual inspection of Figure 4 clearly suggested a difference in connectivity pattern between gain and losses in particular in the amygdala area we performed a further exploratory ROI analysis. However, no significant differences were observed between both conditions in the right or left amygdala region.

\section{DISCUSSION}

Applying a recently developed functional connectivity procedure (Rissman et al., 2004) in an event-related fMRI experiment featuring monetary rewards and losses, we examined whether the interregional interactions maintained by the ventral striatum (NAcc) can be used to characterize and, possibly, dissociate the processing of gains and losses. Standard univariate fMRI analysis revealed a very similar neural network for processing of gains and losses, except for a larger activation observed in the ventral striatum in gain trials. This pattern agrees with previous fMRI studies that underscored the fundamental role of this neural network in the encoding, updating, and maintenance of rewards and punishments (Breiter et al., 2001; Delgado et al., 2000, 2003; Knutson et al., 2001, 2003; McClure et al., 2004; Riba et al., 2008; Tom et al., 2007; Yacubian et al., 2006). 


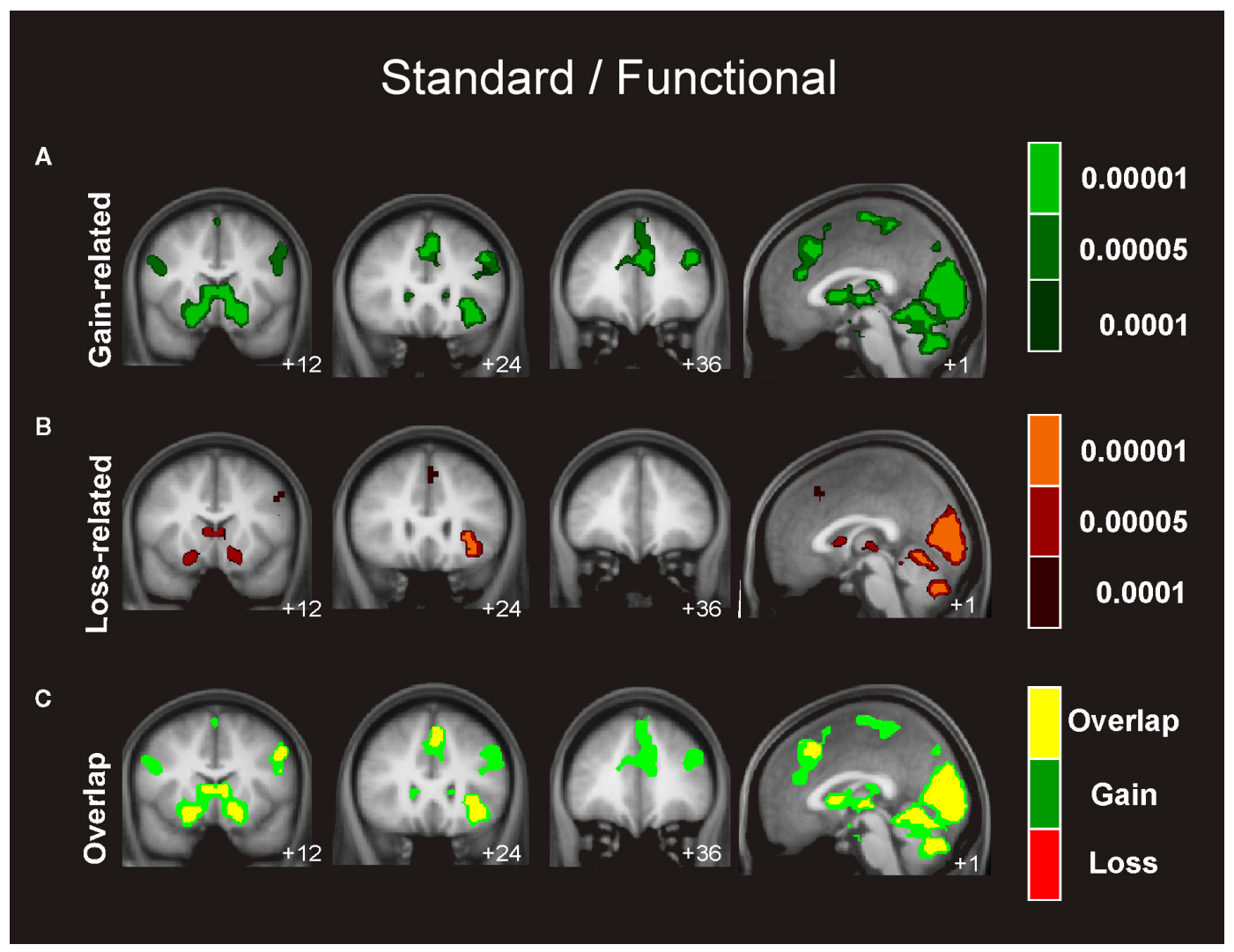

FIGURE 2 | Univariate fMRI analysis in the standard trials. Coronal and sagittal views of group average whole-brain univariate functional analysis superimposed on a group-averaged structural MRI image in standard stereotactic space (T-score overlays). Shown are the gain vs. fixation contrast
(A) and the loss vs. fixation contrast (B) using different statistical thresholds $(P<0.0001 ; P<0.0005 ; P<0.001)$. Gain and loss patterns are simultaneously represented in (C): gain (green, $P<0.001$ ), loss (red, $P<0.001$ ), and conjunction gain $\cap$ loss (yellow, $P<0.001$ and $P<0.001$ ).

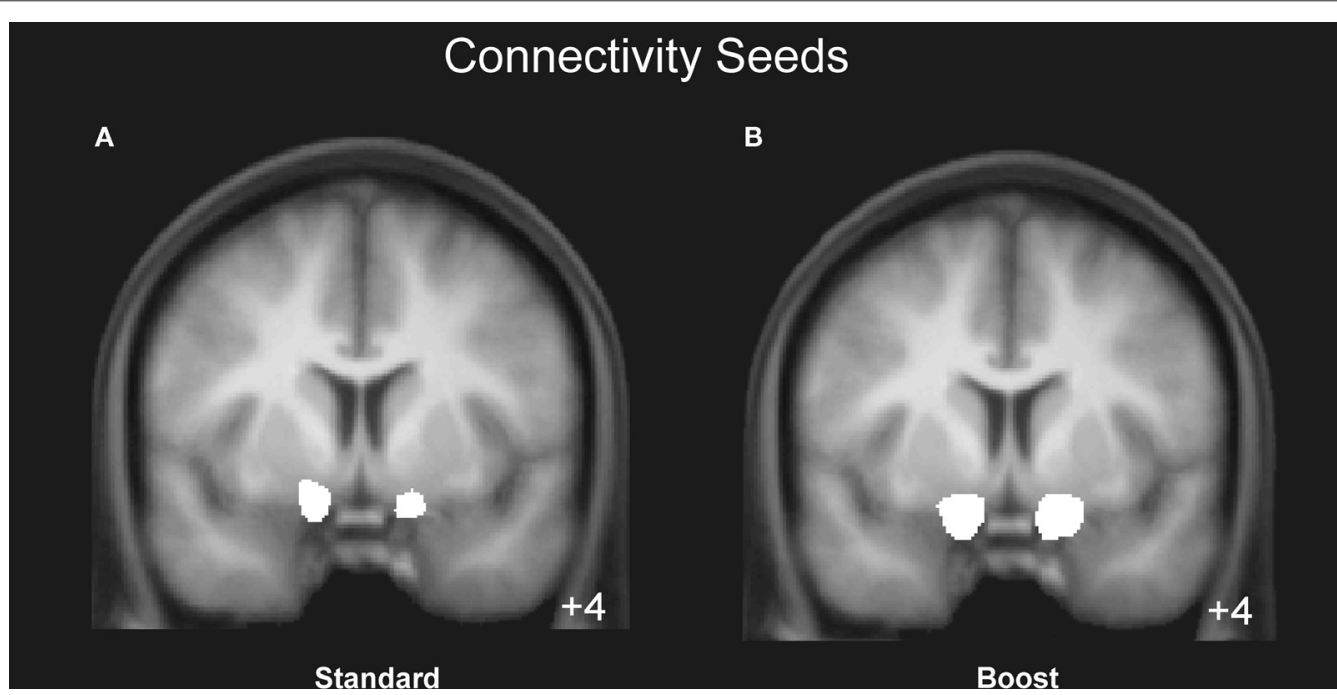

FIGURE 3 | Mean region of interest for the boost and standard condition. The left and right NAcc ROI was functionally defined for each participant in native space by identifying the statistically significant activation cluster in the Gain $(5+25)$ vs. Loss $(5+25)$ and in the Gain (125) vs. Loss (125) contrasts. Displayed is the mean region of interest after normalizing and superimposing the individual ROls in the standard (A) and boost (B) condition on a group-averaged structural MRI image in standard stereotactic space. 
Table 3 | Main effects for valence in the interregional interactions with the ventral striatum for boost trials.

Stereotactic coordinates

\begin{tabular}{llllll}
\hline BA & $X$ & $Y$ & $Z$ & $T$ peak
\end{tabular}

Reward-related interregional interactions

with the ventral striatum in boost trials

$x$
20

32

32
-44

32

$-36$

L hippocampus

R INS

L thalamus

$\mathrm{R}$ thalamus

L MTG

L OIG

R OIG

SN/TA

LVLPC

RVLPC

$R$ ventral striatum

$\begin{array}{rrr} & 20 & -16 \\ & 32 & -64 \\ & -44 & -50 \\ & 32 & -24 \\ & -36 & -16 \\ & 40 & -12 \\ & -8 & -4 \\ 11 & 8 & -8 \\ 19 & -48 & -36 \\ & -44 & -68 \\ 11 & -36 & -92 \\ 11 & -12 & -20 \\ 11 & -24 & 28 \\ & -32 & 40 \\ & 20 & 32\end{array}$

$\begin{array}{rc}-20 & 7.19 \\ -32 & 7.32 \\ -32 & 6.61 \\ -4 & 7.29 \\ -12 & 5.76 \\ -4 & 7.70 \\ 0 & 6.04 \\ 4 & 5.78 \\ -16 & 7.16 \\ -8 & 5.42 \\ -12 & 7.36 \\ -8 & 5.68 \\ -12 & 7.86 \\ -12 & 7.18 \\ -8 & 6.9 \\ -16 & 7.04\end{array}$

\section{LOSS-RELATED INTERREGIONAL INTERACTIONS}

\author{
L subgenual ACC/OFC \\ R subgenual ACC/OFC \\ LAmy \\ R Amy \\ $\mathrm{L}$ cerebellum \\ L hippocampus \\ L MTG
}

\section{R MTG}

$\mathrm{R}$ thalamus

$\mathrm{L}$ thalamus

R INS

SN/TA

$R$ ventral striatum

$L$ ventral striatum

$\begin{array}{rrr}34 / 10 & -12 & 44 \\ 34 / 10 & 12 & 40 \\ & -24 & 4 \\ & 28 & -52 \\ & -8 & -24 \\ 21 & -28 & -52 \\ 21 & -52 & 4 \\ & -52 & -40 \\ 21 & -32 & -12 \\ & 52 & -16 \\ & 16 & -20 \\ & -12 & -24 \\ & 48 & -20 \\ & -12 & 16 \\ & 20 & 4 \\ & -8 & \end{array}$

$-16$

\begin{tabular}{rrr}
44 & -8 & 7.85 \\
40 & -8 & 7.62 \\
4 & -24 & 7.71 \\
8 & -16 & 7.02 \\
-52 & -40 & 7.02 \\
-24 & -24 & 8.52 \\
-52 & 0 & 8.65 \\
4 & -20 & 7.98 \\
-40 & -16 & 4.86 \\
-12 & -8 & 8.28 \\
-16 & 4 & 6.59 \\
-20 & 0 & 6.99 \\
-24 & 0 & 7.45 \\
-20 & -7 & 5.68 \\
16 & -8 & 8.04 \\
4 & -4 & 6.97 \\
& & \\
24 & -16 & 3.12 \\
\hline
\end{tabular}

LOSS VS. GAIN INTERREGIONAL INTERACTIONS

Medial OFC

11

MNI coordinates and T-value for the peak location in a particular identified anatomical cluster. $P<0.0001 ; 20$ voxels spatial extent uncorrected for multiple comparisons. Reported the P-value FWE-corrected at voxel-level. In the loss vs. gain contrast $P<0.001$ uncorrected for multiple comparisons. BA, approximate Brodmann's area; $L$, left hemisphere; $R$, right hemisphere; VLPC, ventral lateral prefrontal cortex; MTG, medial temporal gyrus; INS, insular cortex; ACC, anterior cingulate cortex; Amy, amygdala; SN/TA, substantia nigra/ventral tegmental area.

The functional connectivity analysis using the ventral striatum as a seed region revealed a topographically distinct subcortical-limbicanterior prefrontal network when compared to the previous standard fMRI analysis. Whereas the general connectivity patterns for gain and loss trials showed similarities, there were also remarkable differences. First, the correlation between ventral striatum and the OFC was stronger in loss trials than in gain trials for the boost condition. Second, the amygdala cluster that showed significant correlations to the ventral striatum seed region was considerably larger for losses compared to gains. As this result was not significant, it awaits confirmation before a differential functional connectivity between both regions during the processing of gains and losses can be concluded. In a more general way, the different neural network patterns observed between the standard analysis and the connectivity analysis stress the importance of studying functional connectivity as a complementary tool, as it has been successfully demonstrated in previous studies 
Table 4 | Main effects for valence in the interregional interactions with the ventral striatum in the standard condition.

\begin{tabular}{|c|c|c|c|c|c|}
\hline \multirow{2}{*}{$\begin{array}{l}\text { Reward-related interregional interactions } \\
\text { with the ventral striatum in standard trials }\end{array}$} & \multirow[b]{2}{*}{$\sim \mathrm{BA}$} & \multicolumn{4}{|c|}{ Stereotactic coordinates } \\
\hline & & $x$ & $\boldsymbol{Y}$ & $Z$ & $T$ peak \\
\hline R Amy & & 20 & 0 & -20 & 7.18 \\
\hline L Amy & & -15 & 0 & -20 & 6.86 \\
\hline LINS & & -52 & 8 & -12 & 5.99 \\
\hline LVLPC & $11 / 47$ & -28 & 28 & -12 & 6.37 \\
\hline R ventral striatum & & 16 & 12 & -16 & 5.51 \\
\hline L ventral striatum & & -4 & 8 & -8 & 5.16 \\
\hline \multicolumn{6}{|c|}{ LOSS-RELATED INTERREGIONAL INTERACTIONS } \\
\hline R Amy & & 16 & -4 & -16 & 10.86 \\
\hline L INS & & -60 & 8 & 0 & 6.88 \\
\hline OFC & $10 / 11$ & 12 & 48 & 8 & 8.61 \\
\hline LVLPC & 47 & -28 & 32 & -12 & 7.91 \\
\hline R ventral striatum & & 24 & 12 & -12 & 5.95 \\
\hline L ventral striatum & & -20 & 8 & -12 & 5.13 \\
\hline
\end{tabular}

MNI coordinates and T-value for the peak location in a particular identified anatomical cluster. P<0.0001; 20 voxels spatial extent uncorrected for multiple comparisons. Reported the P-value FWE-corrected at voxel-level. In the loss vs. gain contrast $P<0.001$ uncorrected for multiple comparisons. BA, approximate Brodmann's area; $L$, left hemisphere; $R$, right hemisphere; VLPC, ventral lateral prefrontal cortex; DLPC, dorso lateral prefrontal cortex; INS, insular cortex; ACC, anterior cingulate cortex; Amy, amygdala; OFC, orbitofrontal cortex.

(Buchsbaum et al., 2005; Fiebach and Schubotz, 2006; Gazzaley et al., 2004; Ranganath et al., 2005; Rissman et al., 2004).

\section{FUNCTIONAL CONNECTIVITY ANALYSIS OF GAINS AND LOSSES}

Connectivity analysis revealed a network including the OFC, the insular cortex, the amygdala, and the hippocampus that correlated with the activity observed in the seed region (ventral striatum) in the processing of gains and losses. Following seminal work by Alexander et al. (1986) a number of frontal-basal ganglia circuits dedicated to learning, motivation and motor action selection have been described which modulate cortical processing (Kelley, 2004; Lisman and Grace, 2005; Münte et al., 2008). It has to be pointed out that the functional loops that have been described for these different domains overlap and share some important processing stations. Thus, a more realistic view might be that of a network, as depicted in Figure 7 following earlier work by Kelley (2004). In this figure we highlighted those areas of the learning/motivation/motor preparation network that comprised the network processing gains and losses as revealed by our connectivity analysis. In the following we discuss the role of the different players within this network for the processing of gains and losses.

\section{Amygdala and hippocampus}

A main result of the experience of positive (monetary gains in our experiment) and negative (losses) events is the modulation of future behavior on the basis of learned stimulus-outcome contingencies. Interestingly, the activation of the substantia nigra (SN)/VTA and the hippocampus has been associated with novelty processing and facilitation of memory formation (Schott et al., 2004, 2006; Wittmann et al., 2008). This recent work in humans dovetails nicely with a proposal by Lisman and Grace (2005) that envisions the hippocampus and the midbrain dopaminergic neurons as parts of a functional loop. Novel information that is not yet stored in long-term memory activates the hippocampus which projects to the subiculum, the NAcc, ventral pallidum and to the midbrain, leading to novelty-dependent firing of these cells. This firing evokes a release of DA that enhances long-term potentiation in the hippocampus and as a result the system is learning. In light of the Lisman and Grace account the interregional connectivity between the NAcc and the hippocampus probably reflects the integration of contextual aspects related to reward processing (Adcock et al., 2006; Moore and Price, 1999; Wittmann et al., 2005).

As no significant differences were observed in the amygdala's connectivity to the striatum between gain and loss trials, the numerically greater proportion of activated voxels in the loss trials should be interpreted with caution. The amygdala has traditionally been associated with the processing of aversive states (Baxter and Murray, 2001; Yacubian et al., 2006) but others have also proposed a central role for this structure in processing both aversive and pleasant stimuli (Hamann et al., 1999; Phan et al., 2004; Phelps 




FIGURE 4 | Connectivity analysis for the boost trials. Coronal and axial views of the group average whole-brain interregional interactions with the ventral striatum superimposed on a group-averaged structural MRI image in standard stereotactic space ( $T$-score overlays). Functional connectivity is examined in the reward (A), and punishment (B) condition using different statistical thresholds $(P<0.0001 ; P<0.0005 ; P<0.001)$. Gains and losses connectivity patterns are simultaneously depicted in (C): gain (green, $P<0.001$ ), loss (red, $P<0.001$ ), and conjunction gain $\cap$ loss (yellow, $P<0.001$ and $P<0.001$ ). and LeDoux, 2005; Salinas and White, 1998). It is interesting to note that Yacubian et al. (2006) on the basis of standard activation contrasts distinguished two systems involved in predictions of gains on the one hand and losses on the other hand. In their account the amygdala is involved in the prediction of possible losses whereas the ventral striatum is involved in the prediction of gains. While we examined reward/loss delivery rather than prediction, our results likewise hint at a greater role of the amygdala in the processing of losses than in the processing of gains. These differences appear to be qualitative rather than quantitative in the present task, however. Previous studies have indicated that the OFC-amygdala circuit is critical for the adaptation to changes in stimulus-reward or response-reward mappings (Cools et al., 2004; Goto and Grace, 2005; Kesner and Rogers, 2004; Kringelbach, 2005). Such adaptation processes seem to be more crucial after loss-events than gain-events, because the former indicate the need for a change in behavior.

\section{The insular cortex}

Our results suggest that interregional functional connectivity between the insula and the ventral striatum is equally strong during the processing of gains and losses. This disagrees with earlier findings that the insula is primarily involved in the processing of negative events (Morris et al., 1999; Phillips et al., 1998). Within the context of reward processing, the anterior insula has been related to reward-related uncertainty (Contreras et al., 2007; Ernst et al., 2002; Hsu et al., 2005; Paulus et al., 2003; Preuschoff et al., 2008; Reynolds and Zahm, 2005; Sell et al., 2000) and risk aversion (Kuhnen and Knutson, 2005; Preuschoff and Bossaerts, 2007). In fact, in order to determine the correct value of an uncertain outcome, it is necessary to evaluate the risk, which is then used to estimate the probability of a possible reward (Preuschoff and Bossaerts, 2007). The latter function may explain why this region has also been implicated in drug craving by several imaging studies (Contreras et al., 2007; Garavan et al., 2000; Sell et al., 2000; Wang et al., 1999).

Anatomically, the insular cortex has bidirectional connections with many structures implicated in reward and decision making, including the OFC, the ACC, the NAcc, and the amygdala (Reynolds and Zahm, 2005). Because of its extensive interconnectivity, the insular cortex might be crucial for integrating emotion-related and interoceptive information and to feed this information forward to the OFC and ACC, thus influencing decision making, as well as directly affecting other reward-related limbic regions like the amygdala and NAcc.

\section{The role of the medial OFC in reward processing}

The medial OFC was differentially involved in the processing of loss compared to gain outcomes. The role of the OFC in the 


\section{Standard / Connectivity}

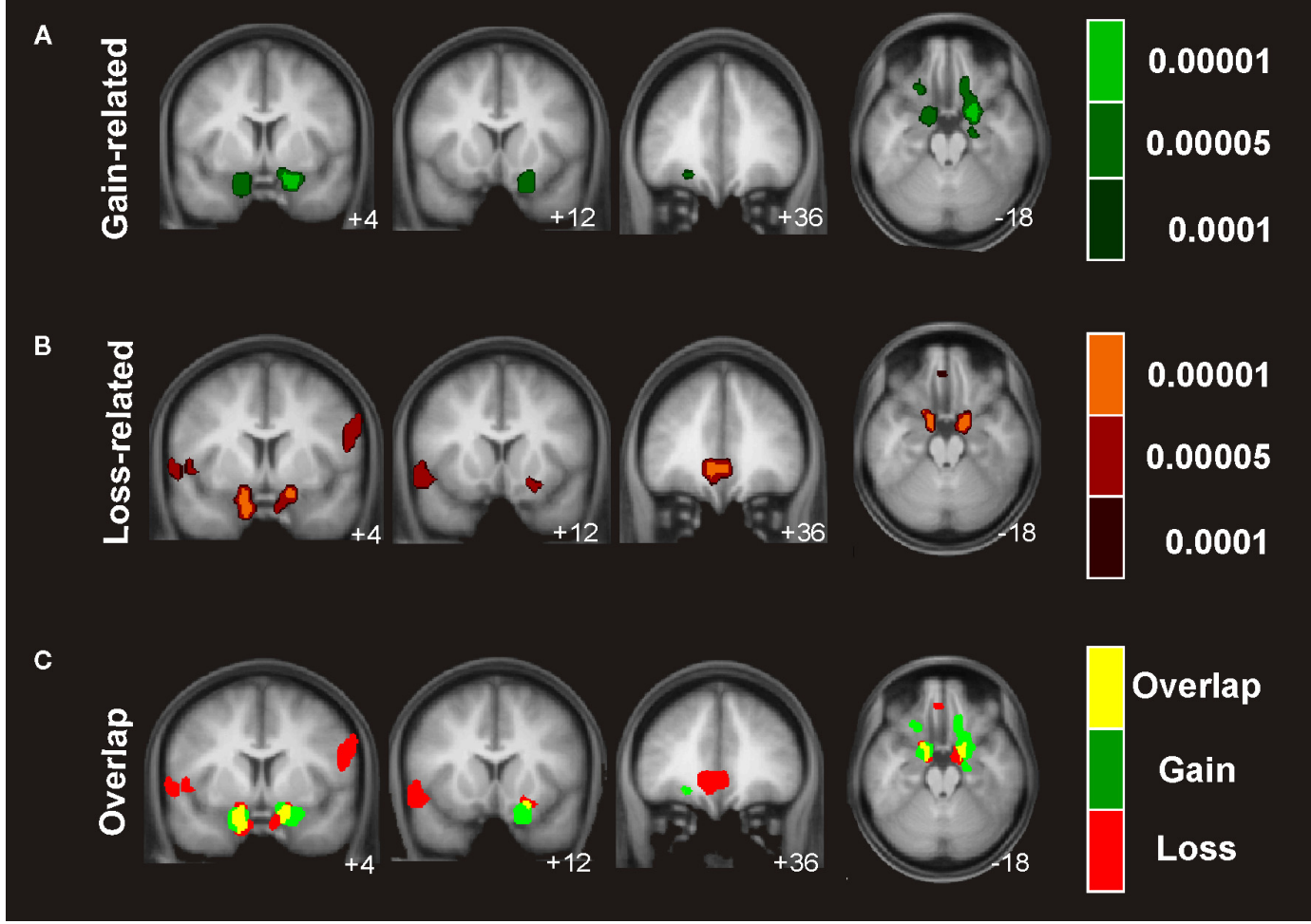

FIGURE 5 | Connectivity analysis for the standard trials. Coronal views and axial of the group average whole-brain interregional interactions with the ventral striatum superimposed on a group-averaged structural MRI image in standard stereotactic space (T-score overlays). Functional connectivity is examined in the reward (A), and punishment (B) condition using different statistical thresholds $(P<0.0001 ; P<0.0005 ; P<0.001)$. Gains and losses connectivity patterns are simultaneously depicted in (C): gain (green, $P<0.001$ ), loss (red, $P<0.001$ ), and conjunction gain $\cap$ loss (yellow, $P<0.001$ and $P<0.001$ ). processing of rewards and punishment has been extensively documented (Rolls, 1996, 2000). O'Doherty et al. (2001) reported an increase in the activity of the medial OFC as a function of the magnitude of the reward or loss incurred. They showed a graded increase in the activation of this region in relation to reward, but also a decrease relative to baseline when a punishment was delivered, a pattern similar to the one documented in Figure 6 for the present study. The deactivation observed in the OFC for loss trials might reflect diminished presynaptic input from NAcc neurons. This idea is supported by reports that NAcc activity is suppressed after reward outcomes (Breiter et al., 2001; Delgado et al., 2000; May et al., 2004) and when anticipated rewards are not obtained (Breiter et al., 2001; Delgado et al., 2000; Knutson et al., 2001, 2003; O’Doherty et al., 2002). Moreover, Yacubian et al. (2006) found higher deactivation in the NAcc during loss trials when the loss condition was less likely to occur and in Tom et al. (2007) activation in both the ventral striatum and the medial OFC decreased as the size of the potential loss increased. This suggests that activation decreases in the NAcc and the medial OFC are related to the impact of a negative outcome. The present study adds the important finding of a stronger functional connectivity observed in losses compared to gains between the OFC and the NAcc. Interestingly, the OFC has recently been studied by a combined functional/structural connectivity approach using a feedback-guided reversal learning task (Cohen et al., 2008). In this study tractography of amygdala-OFC connections predicted an individual's learning behavior following rule reversals, stressing the OFC's role in learning from negative events in concert with the amygdala.

\section{CONCLUSIONS}

In sum, using connectivity analysis with the ventral striatum as a seed region a brain network comprising the amygdala, hippocampus, insular cortex, and OFC was revealed for both boost and standard trials. While the network was similar for both, gain and loss trials, both conditions were only functionally differentiated for the boost trials in the medial OFC for which greater functional connectivity to the ventral striatum was shown in loss trials. Interestingly, a more extended connectivity from the NAcc to the amygdala was observed for loss trials and may reflect that amygdala's role in the implementation of behavioral changes after loss-events.

In light of models mainly based on anatomical and physiological work in animals (c.f. Figure 7) it is interesting to speculate about the information flow within the brain network revealed for the processing of positive and negative feedback events. It appears that, 


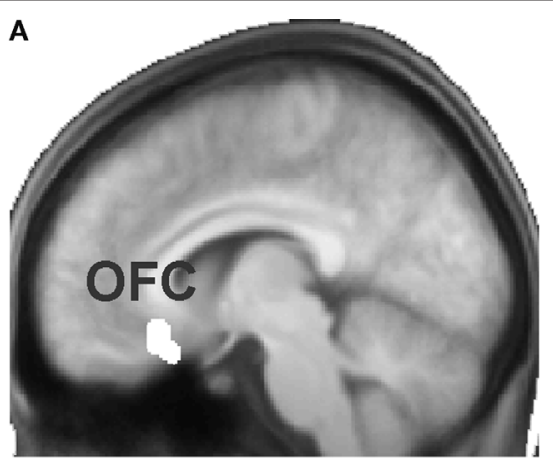

FIGURE 6 | Results for the orbitofrontal cortex. Group-level parameters estimates from the univariate analysis are compared between the gain and the loss condition in the OFC cluster identified in the functional connectivity analysis (contrast between gains and losses). (A)The left panel plots the region of

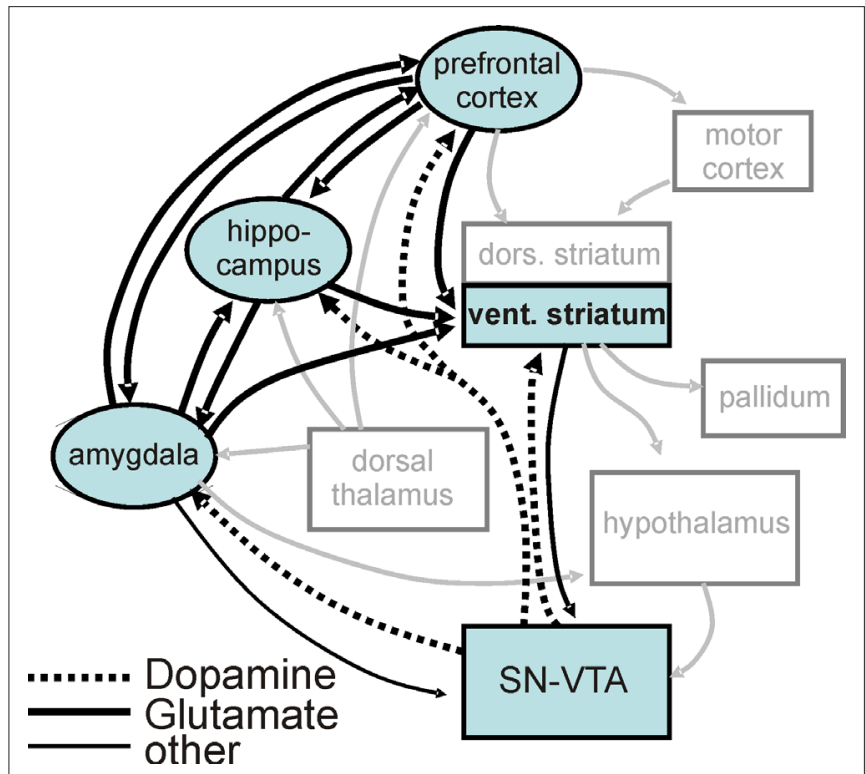

FIGURE 7 | Reward processing network in light of the current results. Illustration of the reward processing network revealed by the current connectivity analysis (black boxes, black arrows) embedded in a wider motivation/learning network (gray boxes and arrows). The wider network is slightly modified (omitting unspecific hypothalamic/thalamic projections) from Kelley (2004).

depending on the particular processing requirements, nodes of the wider motivation/learning/motor action network work together in a flexible manner, leading to situation-specific functional connectivity patterns. Functional connectivity analyses have the unique power to highlight these situation-specific patterns. For example, in a recent study Kahnt et al. (2008) showed that the dorsal and ventral striatum are differentially connected to different midbrain regions tentatively identified as the SN and VTA. Moreover, in this study functional connectivity between the dorsal striatum and the substantia was related to the impact of different feedback stimuli on a participant's future behavior. It is important to bear in mind that spatial overlap between the gain and loss-related networks does not rule out the

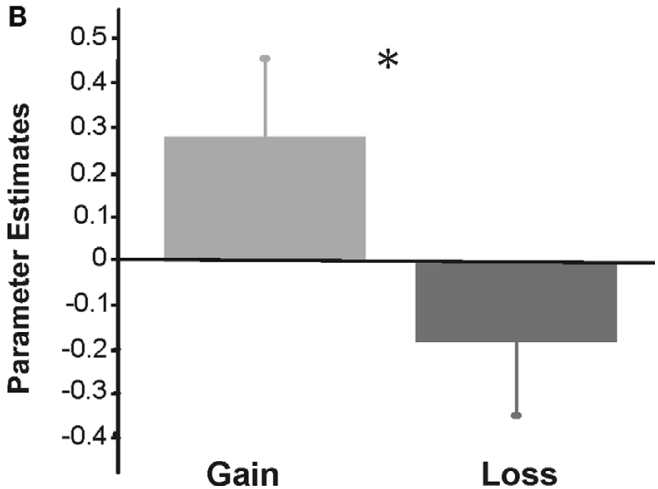

interest selected superimposed in the group-averaged structural MRI image in standard stereotactic space. (B) The right panel shows a bar graph depicting the beta values (general linear model parameter estimates) for gains and losses. Error bars represent standard errors about the mean across subjects.

possibility that different neural populations within the same regions are involved in these different conditions. For example, there is evidence for excitatory and inhibitory cells in the ventral striatum that have functionally different properties. Indeed, Frank et al., (2004) have proposed a differential modulation of these populations during positive and negative reinforcement. These differences can not be disentangled by comparing the functional patterns, since excitatory and inhibitory contributions can not be differentiated in terms of the evoked BOLD response (Logothetis, 2008).

The importance of the dorsal ACC and the ventral striatum in the adjustment of behavior based on the reinforcement learning signals emanating from midbrain regions has been stressed by recent theories (e.g., Holroyd and Coles, 2002). Surprisingly, our functional connectivity analyses with the ventral striatum as a seed did not reveal the dorsal ACC, although anatomical connections do exist and previous studies have reported functional connectivity between them (Cohen et al., 2005; Klein et al., 2007).

Finally, we would like to point out that connectivity analyses are inherently correlational and, thus, without a good anatomical and neurocognitive model no strong statements can be made about the direction of information flow. Coactivity shows that different regions are functionally related, but further experimental manipulations are needed to address the nature of this functional relationship.

\section{ACKNOWLEDGEMENTS}

This work was supported by grants from the Spanish Government (Ramon y Cajal, SEJ2005-06067/PSIC), and the Generalitat de Catalunya (SGR2005-00831) to Antoni Rodriguez-Fornells. Thomas F. Münte is supported by the DFG (SFB 779/TP A5) and the German Government (BMBF contracts 01GW0551 and 01GO0202).

\section{REFERENCES}

Abler, B., Erk, S., Herwig, U., and Walter, H. (2007). Anticipation of aversive stimuli activates extended amygdala in unipolar depression. J. Psychiatr. Res. 41, 511-522.

Adcock, R. A., Thangavel, A., Whitfield-Gabrieli, S., Knutson, B., and Gabrieli, J. D. (2006). Reward-motivated learning: mesolimbic activation precedes memory formation. Neuron 50, 507-517. 
Alexander, G. E., DeLong, M. R., and Strick, P. L. (1986). Parallel organization of functionally segregated circuits linking basal ganglia and cortex. Annu. Rev. Neurosci. 9, 357-381.

Ashburner, J., and Friston, K. J. (1999). Nonlinear spatial normalization using basis functions. Hum. Brain Mapp. 7, 254-266.

Baxter, M. G., and Murray, E. A. (2001). Effects of hippocampal lesions on delayed nonmatching-to-sample in monkeys: a reply to Zola and Squire (2001). Hippocampus 11, 201-203.

Berridge, K. C., and Robinson, T. E. (1998). What is the role of dopamine in reward: hedonic impact, reward learning, or incentive salience? Brain Res. Brain Res. Rev. 28, 309-369.

Breiter, H. C., Aharon, I., Kahneman, D., Dale, A., and Shizgal, P. (2001). Functional imaging of neural responses to expectancy and experience of monetary gains and losses. Neuron 30, 619-639.

Buchsbaum, B., Pickell, B.,Love, T., Hatrak, M., Bellugi, U., and Hickok, G. (2005). Neural substrates for verbal working memory in deaf signers: fMRI study and lesion case report. Brain Lang. 95, 265-272.

Cohen, M. X., Elger, C. E., and Weber, B. (2008).Amygdala tractography predicts functional connectivity and learning during feedback-guided decisionmaking. Neuroimage 39, 1396-1407.

Cohen, M. X., Heller, A. S., and Ranganath, C. (2005). Functional connectivity with anterior cingulate and orbitofrontal cortices during decision-making. Brain Res. Cogn. Brain Res. 23, 61-70.

Contreras, M., Ceric, F., and Torrealba, F. (2007). Inactivation of the interoceptive insula disrupts drug craving and malaise induced by lithium. Science 318, 655-658.

Cools, R., Clark, L., and Robbins, T. W. (2004). Differential responses in human striatum and prefrontal cortex to changes in object and rule relevance. J. Neurosci. 24, 1129-1135.

Daw, N. D., O’Doherty, J. P., Dayan, P., Seymour, B., and Dolan, R. J. (2006). Cortical substrates for exploratory decisions in humans. Nature 441, 876-879.

Delgado,M.R.,Locke,H.M.,Stenger, V. A., and Fiez, J.A. (2003). Dorsal striatum responses to reward and punishment: effects of valence and magnitude manipulations. Cogn. Affect. Behav. Neurosci. 3, 27-38.

Delgado, M. R., Nystrom, L. E., Fissell, C., Noll, D. C., and Fiez, J. A. (2000). Tracking the hemodynamic responses to reward and punishment in the striatum. J. Neurophysiol. 84, 3072-3077.
Dreher,J.C. (2007). Sensitivity of the brain to loss aversion during risky gambles. Trends Cogn. Sci. 11, 270-272.

Ernst, M., Bolla, K., Mouratidis, M., Contoreggi, C., Matochik, J. A., Kurian, V., Cadet, J. L., Kimes, A. S., London, E. D. (2002). Decisionmaking in a risk-taking task: a PET study. Neuropsychopharmacology 26 , 682-691.

Fiebach, C. J., and Schubotz, R. I. (2006). Dynamic anticipatory processing of hierarchical sequential events: a common role for Broca's area and ventral premotor cortex across domains? Cortex 42, 499-502.

Fiorillo, C. D., Tobler, P. N., and Schultz, W. (2003). Discrete coding of reward probability and uncertainty by dopamine neurons. Science 299, 1898-1902.

Frank, M. J., Moustafa, A. A., Haughey, H. M., Curran, T., and Hutchison, K. E. (2007). Genetic triple dissociation reveals multiple roles for dopamine in reinforcement learning. Proc. Natl. Acad. Sci. USA 104, 16311-16316.

Frank, M. J., Seeberger, L. C., and O'reilly, R. C. (2004). By carrot or by stick: cognitive reinforcement learning in parkinsonism. Science 306, 1940-1943.

Friston, K. J., Fletcher, P., Josephs, O., Holmes, A., Rugg, M. D., and Turner, R. (1998). Event-related fMRI: characterizing differential responses. Neuroimage 7, 30-40.

Friston, K. J., Williams, S., Howard, R., Frackowiak, R. S., and Turner, R. (1996). Movement-related effects in fMRI time-series. Magn. Reson. Med. 35, 346-355.

Garavan, H., Pankiewicz, J., Bloom, A., Cho, J. K., Sperry, L., Ross, T. J., Salmeron, B. J., Risinger, R., Kelley, D., and Stein, E. A. (2000). Cue-induced cocaine craving: neuroanatomical specificity for drug users and drug stimuli. Am. J. Psychiatry 157, 1789-1798.

Gazzaley,A., Rissman, J., and Desposito, M. (2004). Functional connectivity during working memory maintenance. Cogn. Affect. Behav. Neurosci. 4, 580-599.

Gehring, W. J., and Willoughby, A. R. (2002). The medial frontal cortex and the rapid processing of monetary gains and losses. Science 295, 2279-2282.

Goto, Y., and Grace, A. A. (2005). Dopaminergic modulation of limbic and cortical drive of nucleus accumbens in goal-directed behavior. Nat. Neurosci. 8, 805-812.

Gottfried, J. A., O’Doherty, J., and Dolan, R. J. (2003). Encoding predictive reward value in human amygdala and orbitofrontal cortex. Science 301, 1104-1107.
Groenewegen, H. J., Wright, C. I., Beijer, A. V., and Voorn, P. (1999). Convergence and segregation of ventral striatal inputs and outputs. Ann. NY Acad. Sci. 877, 49-63.

Hamann, S. B., Ely, T. D., Grafton, S. T., and Kilts, C. D. (1999). Amygdala activity related to enhanced memory for pleasant and aversive stimuli. Nat. Neurosci. 2, 289-293.

Holroyd, C. B., and Coles, M. G. (2002). The neural basis of human error processing: reinforcement learning, dopamine, and the error-related negativity. Psychol. Rev. 109, 679-709.

Hsu, M., Bhatt, M., Adolphs, R., Tranel, D., and Camerer, C. F. (2005). Neural systems responding to degrees of uncertainty in human decision-making Science 310, 1680-1683.

Kahnt, T., Park, S. Q., Cohen, M. X., Beck, A., Heinz, A., and Wrase, J. (2008). Dorsal striatal-midbrain connectivity in humans predicts how reinforcements are used to guide decisions. J. Cogn. Neurosci. [Epub ahead of print]. doi: 10.1162/jocn.2009.21092.

Kelley, A. E. (2004). Memory and addiction: shared neural circuitry and molecular mechanisms. Neuron 44 161-179.

Kelley, A. E., and Berridge, K. C. (2002). The neuroscience of natural rewards: relevance to addictive drugs. $J$. Neurosci. 22, 3306-3311.

Kesner, R. P., and Rogers, J. (2004). An analysis of independence and interactions of brain substrates that subserve multiple attributes, memory systems, and underlying processes. Neurobiol. Learn. Mem. 82, 199-215.

Klein, T. A., Neumann, J., Reuter M., Hennig, J., von Cramon, D. Y., and Ullsperger, M. (2007). Genetically determined differences in learning from errors. Science 318 1642-1645.

Knutson, B., Adams, C. M., Fong, G. W. and Hommer, D. (2001). Anticipation of increasing monetary reward selectively recruits nucleus accumbens. J. Neurosci. 21, RC159.

Knutson, B., Fong, G. W., Bennett, S. M., Adams, C. M., and Hommer, D. (2003). A region of mesial prefrontal cortex tracks monetarily rewarding outcomes: characterization with rapid event-related fMRI. Neuroimage 18, 263-272.

Kringelbach, M. L. (2005). The human orbitofrontal cortex: linking reward to hedonic experience. Nat. Rev. Neurosci. 6, 691-702.

Kuhnen, C. M., and Knutson, B. (2005). The neural basis of financial risk taking. Neuron 47, 763-770.

Lisman, J.E., and Grace, A. A. (2005). The hippocampal-VTA loop: controlling the entry of information into long term memory. Neuron 46, 703-713.

Logothetis, N. K. (2008). What we can do and what we cannot do with $\mathrm{fMRI}$. Nature 453, 869-878.

Marco-Pallares, J., Cucurell, D., Cunillera, T., Garcia, R., AndresPueyo, A., Munte, T.F., and RodriguezFornells, A. (2008). Human oscillatory activity associated to reward processing in a gambling task. Neuropsychologia 46, 241-248.

Marco-Pallares, J., Muller, S. V., and Munte, T. F. (2007). Learning by doing: an fMRI study of feedbackrelated brain activations. Neuroreport 18, 1423-1426.

May, J. C., Delgado, M. R., Dahl, R. E., Stenger, V. A., Ryan, N. D., Fiez, J. A., and Carter, C. S. (2004). Event-related functional magnetic resonance imaging of reward-related brain circuitry in children and adolescents. Biol. Psychiatry 55, 359-366.

McClure, S. M., York, M. K., and Montague, P. R. (2004). The neural substrates of reward processing in humans: the modern role of FMRI. Neuroscientist 10, 260-268.

Moore, C. J., and Price, C. J. (1999). A functional neuroimaging study of the variables that generate categoryspecific object processing differences. Brain 122(Pt 5), 943-962.

Morris, J. S., Scott, S. K., and Dolan, R. J. (1999). Saying it with feeling: neural responses to emotional vocalizations. Neuropsychologia 37, 1155-1163.

Münte, T., Heldmann, M., Hinrichs, H., Marco-Pallares, J., Kramer, U. M., Sturm, V., and Heinze, H. J. (2008). Contribution of subcortical structures to cognition assessed with invasive electrophysiology in humans. Front. Neurosci. 2, 72-78.

Nieuwenhuis, S., Slagter, H. A., von Geusau, N. J. A., Heslenfeld, D. J., and Holroyd, C. B. (2005). Knowing good from bad: differential activation of human cortical areas by positive and negative outcomes. Eur. J. Neurosci.21, 3161-3168.

O’Doherty, J., Kringelbach, M. L., Rolls, E. T., Hornak, J., and Andrews, C. (2001) Abstract reward and punishment representations in the human orbitofrontal cortex. Nat. Neurosci. 4, 95-102.

O’Doherty, J. P., Deichmann, R., Critchley, H. D., and Dolan, R. J. (2002). Neural responses during anticipation of a primary taste reward. Neuron 33, 815-826.

Paulus, M. P., Rogalsky, C., Simmons, A., Feinstein, J.S., and Stein, M. B. (2003). Increased activation in the right insula during risk-taking decision making is related to harm avoidance and neuroticism. Neuroimage 19, 1439-1448. 
Phan, K. L., Wager, T. D., Taylor, S. F., and Liberzon, I. (2004). Functional neuroimaging studies of human emotions. CNS Spectr. 9, 258-266.

Phelps, E. A., and LeDoux, J. E. (2005). Contributions of the amygdala to emotion processing: from animal models to human behavior. Neuron 48, 175-187.

Phillips, M. L., Young, A. W., Scott, S. K., Calder, A. J., Andrew, C., Giampietro, V., Williams, S. C., Bullmore, E. T., Brammer, M., and Gray, J. A. (1998). Neural responses to facial and vocal expressions of fear and disgust. Proc. Biol. Sci. 265, 1809-1817.

Preuschoff, K., and Bossaerts, P. (2007). Adding prediction risk to the theory of reward learning. Ann. NYAcad. Sci. 1104, 135-146.

Preuschoff, K., Quartz, S. R., and Bossaerts, P. (2008). Human insula activation reflects risk prediction errors as well as risk. J. Neurosci. 28, 2745-2752.

Ranganath, C., Heller, A., Cohen, M. X., Brozinsky, C. J., and Rissman, J. (2005). Functional connectivity with the hippocampus during successful memory formation. Hippocampus 15, 997-1005.

Reynolds, S. M., and Zahm, D. S. (2005). Specificity in the projections of prefrontal and insular cortex to ventral striatopallidum and the extended amygdala. J. Neurosci. 25, 11757-11767.

Riba, J., Kramer, U. M., Heldmann, M., Richter, S., and Munte, T. F. (2008). Dopamine agonist increases risk taking but blunts reward-related brain activity. PLoS ONE 3, e2479.
Rissman, J., Gazzaley, A., and D’Esposito, M. (2004). Measuring functional connectivity during distinct stages of a cognitive task. Neuroimage 23, 752-763.

Rolls, E. T. (1996). The orbitofrontal cortex. Philos. Trans. R. Soc. Lond., B, Biol. Sci. 351, 1433-1443.

Rolls, E. T. (2000). The orbitofrontal cortex and reward. Cereb. Cortex 10, 284-294.

Salinas, J. A., and White, N. M. (1998). Contributions of the hippocampus, amygdala, and dorsal striatum to the response elicited by reward reduction. Behav. Neurosci. 112, 812-826.

Schott, B. H., Richardson-Klavehn, A., Henson, R. N., Becker, C., Heinze, H. J., and Duzel,E. (2006). Neuroanatomical dissociation of encoding processes related to priming and explicit memory. J. Neurosci. 26, 792-800.

Schott, B. H., Sellner, D. B., Lauer, C. J., Habib, R., Frey, J. U., Guderian, S., Heinze, H. J., and Duzel, E. (2004). Activation of midbrain structures by associative novelty and the formation of explicit memory in humans. Learn. Mem. 11, 383-387.

Schultz, W. (2007). Behavioral dopamine signals. Trends Neurosci. 30, 203-210.

Schultz, W., Dayan, P., and Montague, P. R. (1997). A neural substrate of prediction and reward. Science 275, 1593-1599.

Sell, L. A., Morris, J. S., Bearn, J., Frackowiak, R. S., Friston, K. J., and Dolan, R. J. (2000). Neural responses associated with cue evoked emotional states and heroin in opiate addicts. Drug Alcohol Depend. 60, 207-216.
Tom, S. M., Fox, C. R., Trepel, C., and Poldrack, R. A. (2007). The neural basis of loss aversion in decisionmaking under risk. Science 315, 515-518.

van Veen, V., Holroyd, C. B., Cohen, J. D. Stenger, V.A., and Carter, C. S. (2004). Errors without conflict: implications for performance monitoring theories of anterior cingulate cortex. Brain Cogn. 56, 267-276.

Voorn, P., Jorritsma-Byham, B., Van, D. C., and Buijs, R. M. (1986). The dopaminergic innervation of the ventral striatum in the rat: a light- and electron-microscopical study with antibodies against dopamine. J. Comp. Neurol. 251, 84-99.

Wang, G. J., Volkow, N. D., Fowler, J. S., Cervany, P., Hitzemann, R. J., Pappas, N. R., Wong, C. T., and Felder, C. (1999). Regional brain metabolic activation during craving elicited by recall of previous drug experiences. Life Sci. 64, 775-784.

Wittmann, B. C., Schiltz, K., Boehler, C. N., and Duzel, E. (2008). Mesolimbic interaction of emotional valence and reward improves memory formation. Neuropsychologia 46, 1000-1008.

Wittmann, B. C., Schott, B. H. Guderian, S., Frey, J. U., Heinze, H. J., and Duzel, E. (2005). Reward-related fMRI activation of dopaminergic midbrain is associated with enhanced hippocampus-dependent long-term memory formation. Neuron 45 , 459-467.

Wrase, J., Kahnt, T., Schlagenhauf, F., Beck, A., Cohen, M. X., Knutson, B., and Heinz, A. (2007). Different neural systems adjust motor behavior in response to reward and punishment. Neuroimage 36, 1253-1262.

Yacubian, J., Glascher, J., Schroeder, K., Sommer, T., Braus, D. F., and Buchel, C. (2006). Dissociable systems for gainand loss-related value predictions and errors of prediction in the human brain. J. Neurosci. 26, 9530-9537.

Yacubian, J., Sommer, T., Schroeder, K., Gläscher, J., Kalisch, R., Leuenberger, B., Braus, D. F., and Büchel, C. (2007). Gene-gene interaction associated with neural reward sensitivity. Proc. Natl. Acad. Sci. USA 104, 8125-8130.

Conflict of Interest Statement: The authors declare that the research was conducted in the absence of any commercial or financial relationships that could be construed as a potential conflict of interest.

Received: 27 October 2008; paper pending published: 11 November 2008; accepted: 29 December 2008; published online: 16 January 2009.

Citation: Camara E, Rodriguez-Fornells $A$ and Münte TF (2009) Functional connectivity of reward processing in the brain. Front. Hum. Neurosci. (2009) 2:19. doi: 10.3389/neuro.09.019.2008

Copyright (c) 2009 Camara, RodriguezFornells and Münte. This is an open-access article subject to an exclusive license agreement between the authors and the Frontiers Research Foundation, which permits unrestricted use, distribution, and reproduction in any medium, provided the original authors and source are credited. 